\title{
METHOD OF ONCOPLASTIC BREAST RESECTION
}

Background. In women with breast cancer the significant postop lateral deviation of the nipple-areola complex may occur if the partial breast resection with lymphadenectomy was performed through one-line straight incision from the middle axilla to the edge of areola.

Objective. To describe radical oncoplastic breast resection with avoiding of nipple-areola deviation and preservation of the symmetry of breasts.

Methods. The study included 16 women, age 32 to 67 years. Breast cancer at the stage I was found in 5 patients, stage IIA - in 4, stage IIB - in 2, and stage IIIA - in 5 patients. We proposed to use curved (broken line) incision with $120^{\circ}$ angle instead of linear incision. Oncoplastic breast resection began with incision of skin and soft tissues in axilla, along the pectoral muscle downward to lateral contour of breast, and then direction was changed at an angle of $120^{\circ}$ towards areola. The tumor projection was encircled by two semi-oval incisions, and then partial breast resection with axillary lymphadenectomy was done. After morphological confirmation of "clear" surgical margins the wound was sutured along the trajectory of the incision.

Results. All women rated the postop cosmetic result as "good" and "satisfactory". The selected configuration of tissue incisions provided physiological position of the operated breast.

Conclusions. Method of "broken line" incision of skin and soft tissue at an angle of $120^{\circ}$ allows performing the radical breast resection with axillary lymph node dissection and prevents postoperative lateral deviation of nipple-areola, and maintains symmetry of the breasts.

KEY WORDS: breast cancer, oncoplastic resection.

\section{Introduction}

In Ukraine, breast cancer of I-II stages is diagnosed in $76.9 \%$ of patients according to the National Cancer Registry [1]. Thus we can expect increasing the likelihood of organ-sparing operations in patients with breast malignancies.

In the world, breast-conserving surgery (BCS) with postoperative whole breast irradiation is generally accepted as a routine method for most patients with early-stage breast cancer [2]. The proportion of patients treated with BCS has increased from $37 \%$ between 1990 and 1992 to $62 \%$ between 2002 and 2004 [3]. Nowadays $70 \%$ of breast cancer patients are treated with BCS, $27 \%$ with mastectomy alone, and $3 \%$ - with mastectomy plus radiation [4]. BCS of early-stage cancer has been proven to be as equally effective as mastectomy in term of local control, distant disease, breast-cancer-specific and overall survival $[3,5]$. BCS depends on the quadrant and distance from the nipple-areolar complex (NAC). They may require an oncoplastic approach to avoid the nipple or cutaneoglandular retraction with aesthetic sequelae [6].

Address for correspondence: Igor Y. Galaychuk, Medical Department of Oncology and Radiology, I. Ya. HorbachevskyTernopil State Medical University, ternopil, ukraine m. Voli, 1, Ternopil, 46001, Ukraine

Tel.: +380506975723; Fax: 380352268648

E-mail: halaychuk@gmail.com
Objective: to present a method of oncoplastic breast resection with avoiding of nipple-areola deviation and preservation of symmetry of breasts.

\section{Methods}

The study included 16 women, age 32 to 67 years (mean $51.7 \pm 2.5 \mathrm{yr}$.). Breast cancer at the first stage (T1NOM0) was found in 5 patients, stage IIA (T2NOM0, T1N1M0) - in 4, stage IIB (T2N1M0) - in 2, and stage IIIA (T1N2M0) - in 5 patients. Category T1 tumor was diagnosed in 12, and T2 - in 4 patients. In 11 patients the tumor was located in the upper outer quadrant, and in 5 cases - in the inferolateral quadrant.

Staging procedures included: clinical examination of breasts and regional lymph nodes, ultrasound of breasts and ways of lymphatic drainage, mammography, lung X-ray or CT scan, liver ultrasound, fine needle aspiration or core biopsy of tumor, blood analyses and CA-15.3. Histological and biological evaluation results: tumor size, histologic type, grading, margin histology, receptors (ER, PR, Her2neu) and Ki-67 status.

The general scheme of treatment consisted of neoadjuvant chemotherapy, 2-3 cycles (6 patients) or chemoradiation therapy (10 patients). All patients underwent sectoral (or partial) resection of breast that included $2-3 \mathrm{~cm}$ of normal tissue surrounding 
the malignant tumor; also the overlying skin and underlying fascia had to be removed. These radical sectoral resections were performed with axillary lymph nodes dissection by using the suggested oncoplastic technique [7]. In postoperative period the correction of anticancer treatment was done with regard to molecular subtypes of breast cancer. Patients' quality of life was assessed by EORTC scale QLQ-C30 BR23.

Oncoplastic breast resection.

Technical consideration. Radical sectoral resection in breast cancer patients consists of removing of the outer breast sector with simultaneous axillary lymphadenectomy. Cosmetic effects of such operations are not always satisfactory and mostly because resection of the breast and axillary lymph node dissection are performed on one-line tissue incision from the middle axilla to areola (Fig. 1-AC ). Postoperative linear scar causes significant lateral deviation of the areola and nipple (Fig. 1 - D). To avoid deviation and preserve symmetry, surgeon must perform the extra step operation - the centralization of nipple-areola complex (NAC), i.e., medial transposition of the NAC (Fig. 1- $C^{1}$ ). We propose, instead of linear incision (AC), to apply the curved (broken line) incision with $120^{\circ}$ angle between two lines $A B$ and $B C$ (Fig. 1). Because of this incisional geometry, when $A B C>A C$, the postoperative sutures (or scars) have discordant power vectors that provide physiological position of the operated breast.

Clinical case. In patient N., 41 years old, the 10 $\mathrm{mm}$ tumor in upper quadrant of right breast was found by ultrasound and mammographic examination. Fine needle aspiration biopsy confirmed moderately differentiated cancer. Axillary lymph nodes were 18 $\mathrm{mm}$ with margin hyperplasia according to the ultrasound findings. Diagnosis: cancer of the right breast cT1NxM0. Treatment scheme: neoadjuvant chemotherapy (Doxorubicin-Cyclophosphamide, $\times 2$ cycles) and preoperative $\mathrm{Co}^{60}$ external radiation therapy on breast and axilla (4.5-5.0 Gy per fraction,

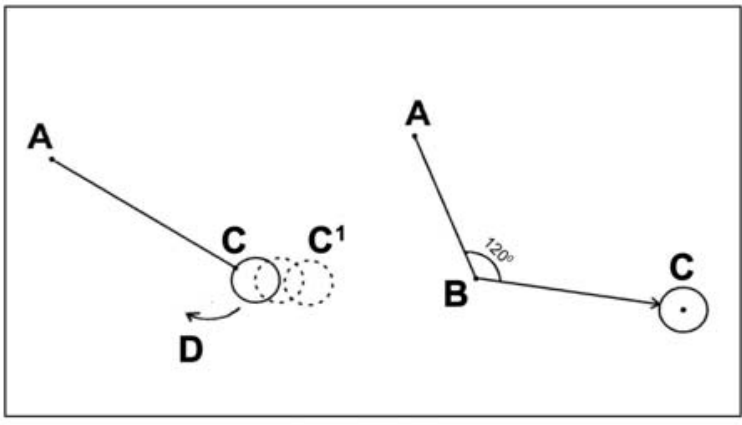

\section{$A C<A B C$}

Fig. 1. Geometry of incisions: A - axilla, C - nipple-areola complex (NAC), B - lateral border of breast, D - direction of deviation, $\mathrm{C}^{1}$ - transposition of NAC.
5 days, doses on axilla 34.2 , on breast 38.2 Gy equivalent); surgery - radical partial breast resection with axillary dissection; postoperative radiation therapy on the breast only (2.2 Gy $\times 10$ days, total dose on breast $60.2 \mathrm{~Gy}$ ) and chemotherapy (Cisplatin-Paclitaxel).

Surgery. The operative designs made in upright position of patient marking tumor's projection on skin with help of breast ultrasound. Oncoplastic breast resection was started with incision of the skin and soft tissues in axilla along the pectoral muscle downward to the lateral contour of breast, and then changed direction at an angle of $120^{\circ}$ towards areola (Fig. 2). The tumor projection was embraced by two semi-oval incisions, and then partial breast resection with axillary lymphadenectomy was done (Fig. 3). After morphological confirmation of "clear" margins in surgical specimen the wound was sewn along the trajectory of the incision (Fig. 4). Surgical specimen: a tumor size of $5 \times 7 \mathrm{~mm}$ at a distance of $2.0 \mathrm{~cm}$ to the margins of resection, with adequate volume ratio "tumor - to - breast tissue". The 11 lymph nodes ranging in size from 5 to $15 \mathrm{~mm}$ were present in axillary fat (Fig. 5). Histology: invasive ductal carcinoma, grade G2; lymph nodes with hyperplasia and angiomatosis; margin tissues histologically negative. Immunohistochemistry: ER-, PR-, Her2-

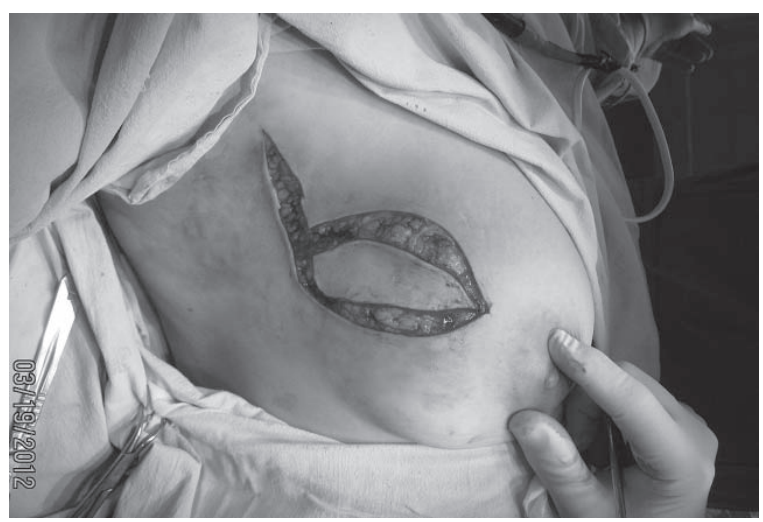

Fig. 2. Clinical case: trajectory of "broken line" incision on axilla and breast.

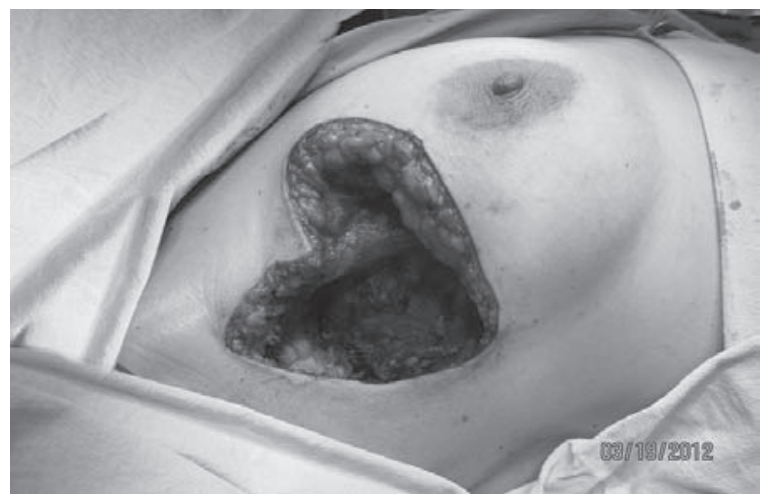

Fig. 3. On-table result of radical breast's resection with axillary lymphadenectomy. 


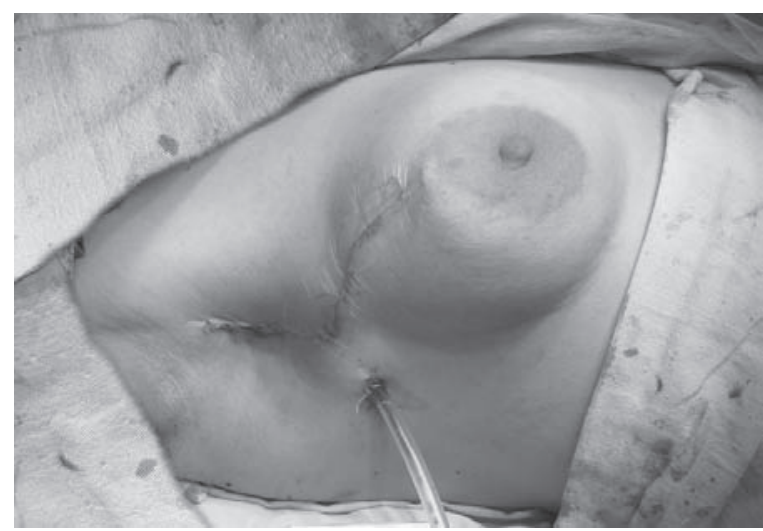

Fig. 4. Completed of oncoplastic breast's resection.

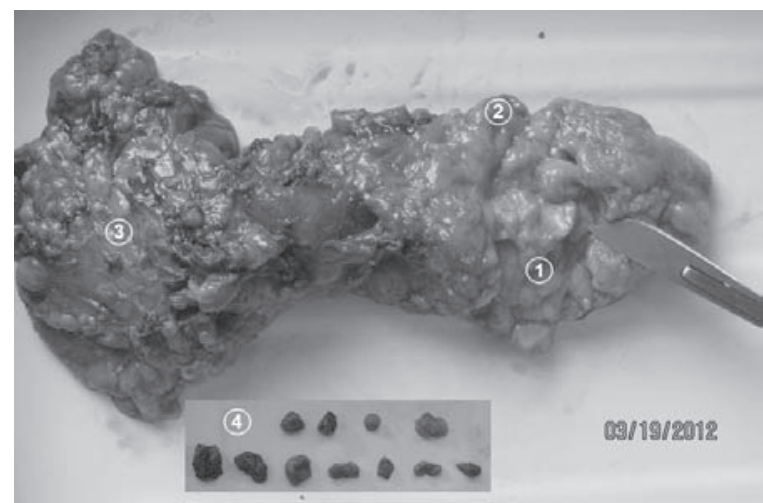

Fig. 5. Surgical specimen (1 - cancer, 2 - breast tissue, 3 axillary fat, 4 - axillary lymph nodes)

neu++. According to the clinical and morphological data, final diagnosis was determined as: cancer of the right breast $p$ T1bNOM0G2, stage I, ER/PR-, Her2-neu++.

In postoperative period wound healing was without any complication. Patient completed treatment by radiation therapy on operated breast and systemic chemotherapy. Two years follow-up with clinical examination, breast ultrasound (every 3 months) and annual X-ray mammography revealed no signs of relapse. Outcome of oncoplastic surgery is satisfactory; breasts are located symmetrically with good cosmetic effect (Fig. 6).
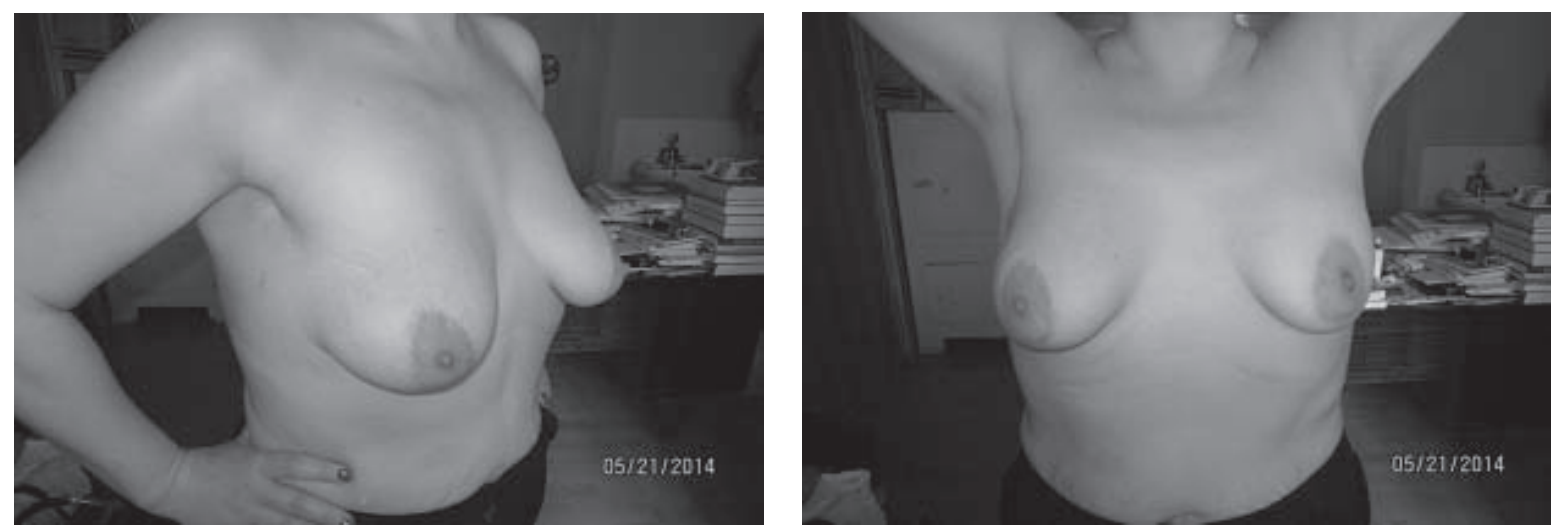

Fig. 6. Cosmetic results 2-years after oncoplastic resection of right breast.

\section{Results}

Complications. Swelling of the operated breast was observed in 11 (69.0\%) women, and it lasted up to 6 months; lymphedema of upper extremity was noted in 4 (25.0\%); seroma around postoperative suture was found in $7(44.0 \%)$ of patients; pain and stiffness in the shoulder joint occurred in $6(38.0 \%)$ women. The postoperative cosmetic result all patients rated as "good" and "satisfactory". The selected configuration of tissue incisions provided a physiological position of the operated breast.

During the 1-4 years of follow-up, 14 patients $(88.0 \%)$ were without evidence of recurrence; one patient died due to metastases into meningeal membranes; juxtaregional lymph node metastases developed in one patient after two years .

The organ-sparing surgery was performed in cases of tumor placing at a short distance from the regional lymph nodes in the outer quadrants of the sufficient developed breasts, when the proportion "tumor-to-breast volume" was more than 1:75-100. It was noted that neoadjuvant anticancer chemoand radiation therapy reduced the volume of primary breast tumor and lymph nodes and by these enabled to do breast-conserving surgery. On the other side the organ-sparing surgery can be safely performed after neoadjuvant chemo- and radiation therapy in patients with breast cancer.

\section{Discussion}

Breast-conservative surgery is considered today as equivalent, if not superior, to mastectomy in terms of breast-cancer-specific survival. As shown in Agarwal et al. analysis of 132,149 women with earlystage invasive breast cancer (tumor $\leq 4 \mathrm{~cm}$; $\leq 3$ positive lymph nodes) that underwent BCS have a higher rate of disease-specific survival than those who undergo mastectomy. The 5-year breast cancer-specific survival rates of patients who underwent BCS, a mastectomy alone, or a mastectomy with radiation were $97 \%, 94 \%$, and $90 \%$, respectively $(p<0.001)[4]$. 
The comparative study of 112,154 eligible women (T1/T2, stages I or II disease) included 61,771 who received lumpectomy (BCS) and radiation, and 50,383 who had mastectomy without radiation was published recently [3]. The $51 \%$ of women under the age of 40 underwent BCS compared with $59 \%$ of women age 50 to 59 years and $51 \%$ of women age 70 to 80 years. The group achieving greatest benefit in overall survival with BCS relative to mastectomy was women at the age of 50 and older who were diagnosed with HR-positive tumors. The smallest benefit was seen among women who were under age of 50 diagnosed with HR-positive tumors. Kaplan-Meier survival estimation showed significantly greater overall and breast cancer-specific survival favoring BCS over mastectomy [3].

In another prospective, randomized study of 749 elderly patients with tumor less than $25 \mathrm{~mm}$ and $\mathrm{pNO}$, $\mathrm{ER}+, \mathrm{G} 1-2, \mathrm{Ki}-67<20 \%$, concluded that whole breast irradiation after BCS can be omitted in selected patient. After 9 years of median follow-up no significant statistical difference was found regarding overall survival and distant disease free survival between both groups with wide resection of the breast tumor (BCS) and BCS plus 50 Gy breast radiotherapy [5].

Thus, these data and our findings provide confidence that BCT remains an effective alternative to mastectomy for early stage disease regardless of age or HR status.

\section{Conclusions}

Method of "broken line" incision of skin and soft tissue at an angle of $120^{\circ}$ allows performing radical breast resection with axillary lymph node dissection and prevents postoperative scar's lateral deviation of nipple-areola, and maintains the symmetry of the breasts.

Suggested method of oncoplastic resection is advisable to apply in the case of lateral localization of tumor in the breast.

Organ-sparing surgery can be safely performed after neoadjuvant chemo- and radiation therapy in patients with breast cancer.

\section{References}

1. National Cancer Registry of Ukraine. Bulletin No. 14. Cancer in Ukraine, 2011-2012. Kyiv; 2013.

2. Veronesi U, Stafyla V, Luini A, Veronesi P. Breast cancer: from "maximum tolerable" to "minimum effective" treatment. Front Oncol 2012; 2: 125-130.

3. Hwang ES, Lichtensztajn DY, Gomez SL, Fowble B, Clarke CA. Survival after lumpectomy and mastectomy for early stage invasive breast cancer. The effect of age and hormone receptor status. Cancer 2013; 119: 1402-1411.

4. Agarwal S, Pappas L, Neumayer L, Kokeny K, Agarwal J. Effect of breast conservation therapy vs mastectomy on disease-specific survival for early-stage breast cancer. JAMA Surg. Available at http://archsurg. jamanetwork.com/article. aspx?articleid $=1813803$
Accessed January 15, 2014.

5. Tinterri C, Gatzemeier W, Costa A, Gentilini MA, Zanini V, Regolo L. et al. Breast-conservative surgery with and without radiotherapy in patients aged 55-75 years with early-stage breast cancer: a prospective, randomized, multicenter trial analysis after 108 months of median follow-up. Ann Surg Oncol 2014; 21: 408-415.

6. Fitoussi A, Berry MG, Couturaud B, Salmon RJ. Oncoplastic and reconstructive surgery for breast cancer. The Institute Curie experience. - Springer-Verlag Berlin Heidelberg; 2009.

7. Patent 86401U UA, A61B 17/00. Method of oncoplastic resection of mammary gland. Galaychuk I.Y. № 2013 08870; Appl. 15.07.13; Publ. 25.12.13. Bulletin № 24. 